\title{
Generation of Primordial Magnetic Fields on Linear Overdensity Scales
}

\author{
Smadar Naoz* and Ramesh Narayan \\ Harvard-Smithsonian Center for Astrophysics, Institute for Theory and Computation, 60 Garden Street, \\ Cambridge, Massachusetts 02138, USA
}

(Received 1 April 2013; revised manuscript received 17 May 2013; published 29 July 2013)

\begin{abstract}
Magnetic fields appear to be present in all galaxies and galaxy clusters. Recent measurements indicate that a weak magnetic field may be present even in the smooth low density intergalactic medium. One explanation for these observations is that a seed magnetic field was generated by some unknown mechanism early in the life of the Universe, and was later amplified by various dynamos in nonlinear objects like galaxies and clusters. We show that a primordial magnetic field is expected to be generated in the early Universe on purely linear scales through vorticity induced by scale-dependent temperature fluctuations, or equivalently, a spatially varying speed of sound of the gas. Residual free electrons left over after recombination tap into this vorticity to generate magnetic field via the Biermann battery process. Although the battery operates even in the absence of any relative velocity between dark matter and gas at the time of recombination, the presence of such a relative velocity modifies the predicted spatial power spectrum of the magnetic field. At redshifts of order a few tens, we estimate a root mean square field strength of order $10^{-25}-10^{-24} \mathrm{G}$ on comoving scales $\sim 10 \mathrm{kpc}$. This field, which is generated purely from linear perturbations, is expected to be amplified significantly after reionization, and to be further boosted by dynamo processes during nonlinear structure formation.
\end{abstract}

DOI: 10.1103/PhysRevLett.111.051303

PACS numbers: 98.62.En, 98.80.- $\mathrm{k}$

Introduction.-Galaxies in the local Universe have coherent magnetic fields with strength $\sim 10^{-6} \mathrm{G}[1-3]$. Similar field strengths are seen in galaxies up to redshift $\sim 2[2,4,5]$. One explanation is that the observed fields originated from primordial magnetic fields which were created in the very early Universe and were later amplified during the formation of the galaxies. Another possibility is that there were no primordial fields and the observed fields were generated spontaneously during the gravitational collapse of galaxies [6,7].

There is independent evidence for a pre-galactic seed magnetic field in the intergalactic medium (IGM). This is based on the lack of detection of inverse Compton $\mathrm{GeV}$ radiation from charged secondaries associated with extragalactic $\mathrm{TeV}$ sources. A magnetic field greater than $\sim 10^{-16} \mathrm{G}$ can deflect secondaries sufficiently to explain the observations $[8,9]$; the required field strength has been reduced to $10^{-18} \mathrm{G}$ in a recent study [10]. Although this evidence for magnetic fields in the IGM emphasizes the notion that the fields are primordial (see Ref. [11]), it is possible that the fields originated by baryonic outflows from already formed galaxies [6,7]. Also the absence of secondary radiation from $\mathrm{TeV}$ sources may have nothing to do with a magnetic field but are the result of beam instabilities which slow down the particles before they can produce significant inverse Compton radiation [12] (see also Ref. [13]). Other recent studies which have considered the influence of primordial magnetic fields on the cosmic microwave background (CMB) and Ly $\alpha$ clouds [14-16] give an upper limit on the present-day large scale magnetic field in the IGM of $\sim 10^{-9} \mathrm{G}$.
In an influential study, Biermann (1950; Ref. [17]) showed that currents must flow whenever a plasma has a rotational vortexlike motion. These currents will lead to the generation of magnetic field starting from zero field. The process has been coined in the literature as the "Biermann battery", and several astrophysical applications have been discussed. These range from the generation of magnetic fields in stars $[17,18]$ to seed magnetic fields on galactic scales [19-23]. The latter studies typically use nonlinear gas-dynamical processes such as those that occur in shocks during structure formation.

It has been argued that magnetic fields at the time of recombination may be generated on large scales $(>600 \mathrm{kpc}$ ) through second-order couplings between photons and electrons [24]. Here we consider smaller scales, and we show that seed magnetic fields can be produced in the early Universe starting from zero field purely as a consequence of the growth of linear overdensities. We consider the evolution of density and temperature fluctuations of the baryonic matter after the time of recombination. We follow the approach described in Ref. [25], where the key new effect that permits the generation of magnetic fields is a spatially varying speed of sound. We also consider the effect of the relative velocities between the dark matter (DM) and baryons at the time of recombination [26]. The latter effect has been shown to have a considerable effect on the evolution of overdensities at high redshifts [26-33]. Here we show that it has a noticeable effect also on the growth of the magnetic field.

Throughout this Letter, we adopt the following cosmological parameters: $\left(\Omega_{\Lambda}, \Omega_{\mathrm{M}}, \Omega_{b}, n, \sigma_{8}, H_{0}\right)=(0.72,0.28$, 0.046, 1, 0.82, and $\left.70 \mathrm{~km} \mathrm{~s}^{-1} \mathrm{Mpc}^{-1}\right)$ [34]. 
Linear evolution of overdensities in the early Universe.After cosmic recombination, the baryonic gas in the Universe decouples mechanically from the photons, but remains thermally coupled down to $z \sim 150$. This coupling is a result of CMB photons scattering off the residual free electrons, which constitute a fraction $\sim 10^{-4}$ of the bound electrons. Even at $z<150$ the baryons still retain some memory of this heating, which induces scale-dependent temperature fluctuations. Naoz and Barkana (2005, Ref. [25]) took this effect into account and computed the linear growth of baryonic density and temperature fluctuations separately. At large wave numbers $\left(k>100 \mathrm{Mpc}^{-1}\right)$ the growth of baryon density fluctuations is changed significantly by the effect of the inhomogeneous sound speed, by up to $30 \%$ at $z=100$ and $10 \%$ at $z=20$. This has an important impact on high- $z$ gas rich halos [35].

It was shown recently that not only are the amplitudes of the DM and baryonic density fluctuations different at early times, so too are their velocities [26]. After recombination, the sound speed of the baryons drops dramatically, while the DM velocity remains high. As a result, the relative velocity of baryons with respect to the DM becomes supersonic. This relative velocity, which is generally referred to as the "stream velocity" in the literature, remains coherent on scales of a few Mpc and is of the order of $\sim 30 \mathrm{~km} \mathrm{~s}^{-1}$ at the time of recombination [26].

For completeness we write here the coupled second order differential equations that govern the evolution of the dimensionless density fluctuations of the DM $\delta_{\mathrm{dm}}$ and of the baryons $\delta_{\mathrm{b}}$ :

$$
\begin{gathered}
\ddot{\delta}_{\mathrm{dm}}+2 H \dot{\delta}_{\mathrm{dm}}-f_{\mathrm{dm}} \frac{2 i}{a} \mathbf{v}_{\mathrm{bc}} \cdot \mathbf{k} \dot{\delta}_{\mathrm{dm}} \\
=\frac{3}{2} H_{0}^{2} \frac{\Omega_{m}}{a^{3}}\left(f_{\mathrm{b}} \delta_{\mathrm{b}}+f_{\mathrm{dm}} \delta_{\mathrm{dm}}\right)+\left(\frac{\mathbf{v}_{\mathrm{bc}} \cdot \mathbf{k}}{a}\right)^{2} \delta_{\mathrm{dm}} \\
\ddot{\delta}_{\mathrm{b}}+2 H \dot{\delta}_{\mathrm{b}}= \\
\frac{3}{2} H_{0}^{2} \frac{\Omega_{m}}{a^{3}}\left(f_{\mathrm{b}} \delta_{\mathrm{b}}+f_{\mathrm{dm}} \delta_{\mathrm{dm}}\right) \\
-\frac{k^{2}}{a^{2}} \frac{k_{B} \bar{T}}{\mu}\left(\delta_{\mathrm{b}}+\delta_{T}\right),
\end{gathered}
$$

where $\Omega_{m}$ is the present-day matter density as a fraction of the critical density, $k$ is the comoving wave number of the perturbation, $\mathbf{v}_{\mathrm{bc}}$ is the relative velocity between the baryons and DM in a local patch of the Universe, $a$ is the scale factor of the Universe, $H_{0}$ is the present day value of the Hubble parameter, $\mu$ is the mean molecular weight of the gas, $\bar{T}$ is the mean temperature of the baryons, $f_{\mathrm{b}}\left(f_{\mathrm{dm}}\right)$ is the cosmic baryonic (DM) fraction and $\delta_{T}$ is the dimensionless baryon temperature fluctuation (see Refs. [26,33] for further discussion of these equations). Derivatives are with respect to the clock time.

The linear evolution of the $\delta_{T}$ may be written down similarly $[25,36]$. Including an additional term due to fluctuations of the electron overdensity $\delta_{e}$ :

$$
\begin{aligned}
\dot{\delta}_{T}= & \frac{2}{3} \dot{\delta}_{\mathrm{b}}+\frac{x_{e}(t)}{t_{\gamma}} a^{-4}\left\{\frac{\bar{T}_{\gamma}}{\bar{T}}\left(\delta_{T_{\gamma}}-\delta_{T}\right)\right. \\
& \left.+\left(\delta_{\gamma}+\delta_{e}\right)\left(\frac{\bar{T}_{\gamma}}{\bar{T}}-1\right)\right\},
\end{aligned}
$$

where $\delta_{\gamma}$ is the photon density fluctuation, $t_{\gamma}^{-1}=8.55 \times$ $10^{-13} \mathrm{yr}^{-1}$, and $\bar{T}_{\gamma}$ and $\delta_{T_{\gamma}}$ are the mean photon temperature and its fluctuation, respectively.

The evolution of the mean free electron fraction $x_{e}$ as a function of time is

$$
\dot{x}_{e}=-\alpha_{B}(T) x_{e}^{2} n_{\mathrm{H}}(1+y),
$$

where $\alpha_{B}(T)$ is the case $B$ recombination coefficient as a function of the gas temperature, $n_{\mathrm{H}}$ is the total hydrogen number density, and $y=n_{\mathrm{He}} / n_{\mathrm{H}}$ where $n_{\mathrm{He}}$ is the helium number density. Fluctuations in the electron density, $\delta_{e}=\Delta n_{e} / n_{e}=\Delta x_{e} / x_{e}$, evolve according as

$$
\dot{\delta}_{e}=-\alpha_{B}(T)(1+y) x_{e} n_{\mathrm{H}}\left(\delta_{b}+\delta_{e}\right) .
$$

We show below that the magnetic field grows because of the presence of the residual free electrons. It is highly sensitive to the evolution of $\delta_{e}$, but not to the actual electron number density.

Equation (3) describes the evolution of the gas temperature in the postrecombination era but before the formation of the first galaxies, when the only external heating arises from Compton scattering of the remaining free electrons on the CMB photons [25]. The first term in Eq. (3) describes the adiabatic cooling of the gas, while the second term is the result of Compton interactions. An important effect of this equation is that it introduces a scale dependent behavior in the fluctuations of the temperature, free electron density and baryon density. In this full thermal evolution calculation, the sound speed $\left(c_{s}^{2}=d p / d \rho\right.$, where $p$ is the pressure of the gas), varies spatially, simply because $\delta_{\mathrm{b}}$ and $\delta_{T}$ have the following relation

$$
1+\frac{\delta_{T}}{\delta_{\mathrm{b}}}=\frac{c_{s}^{2}}{k_{B} \bar{T} / \mu}=\gamma_{\mathrm{eff}},
$$

where $\gamma_{\text {eff }}$ is a scale dependent, effective adiabatic index.

In Fig. 1 we show the ratios $\delta_{T} / \delta_{b}$ (top panel) and $\delta_{T} / \delta_{e}$ (bottom panel) as a function of $k$. At the largest scales (smallest $k$ ), the baryons follow the DM density, and $\delta_{T} / \delta_{b}$ evolves from $1 / 3$ (at high redshift where the baryons are tightly coupled to the relativistic CMB) to $\sim 2 / 3$ (lower redshift where the baryons expand adiabatically as an independent nonrelativistic fluid). Considering first the zero stream velocity case, small scales (large $k$ ) at high redshift show Jeans scale oscillations which are suppressed at lower redshift (there is only a slight minimum for $z=30$ ). For $v_{\mathrm{bc}}=1 \sigma_{\mathrm{vbc}}$, the small scale baryon fluctuations drop, and are less important compared to the Compton heating [see Eq. (3)] which results in a slight increase of $\delta_{T}$ (compared to the zero stream velocity). 


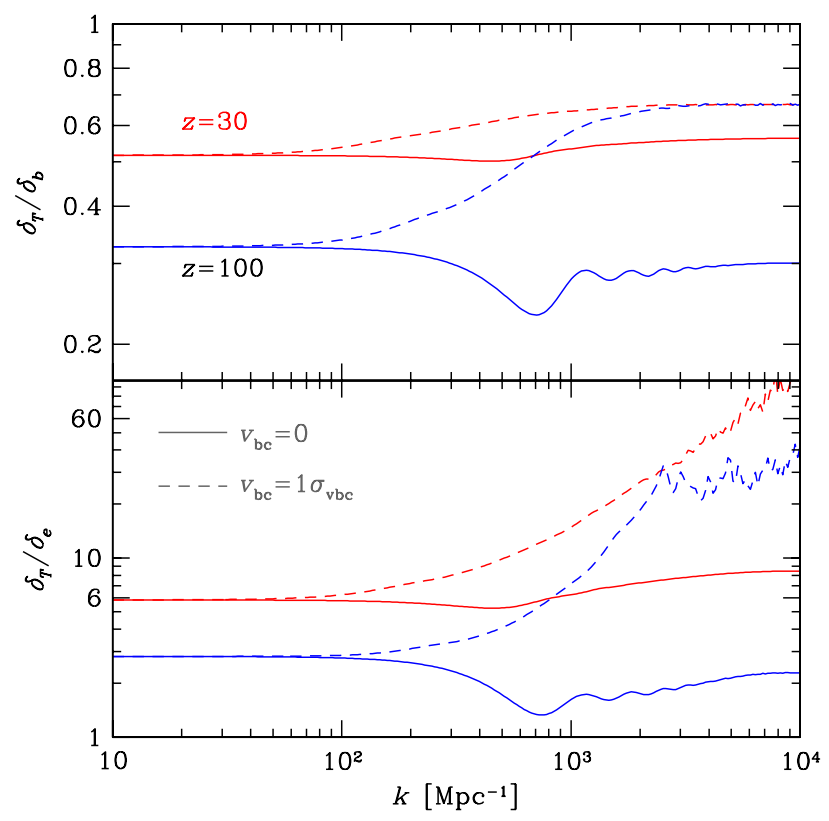

FIG. 1 (color online). Perturbation ratios $\delta_{T} / \delta_{b}$ (top panel) and $\delta_{T} / \delta_{e}$ (bottom panel) as a function of wave number $k$. We consider two cases: no stream velocity, $v_{\mathrm{bc}}=0$ (solid lines), and a typical stream velocity, $v_{\mathrm{bc}}=1 \sigma_{\mathrm{vbc}}$ (dashed lines). Results are shown for two redshifts, $z=100$ (blue lines) and $z=30$ (red lines).

These two effects result in an increase of the ratio $\delta_{T} / \delta_{b}$ as a function of scale. The free electron fluctuations are further suppressed in the case of $v_{\mathrm{bc}}=1 \sigma_{\mathrm{vbc}}$ compared to the case of zero stream velocity which results in a larger increase in the ratio $\delta_{T} / \delta_{e}$.

Biermann battery in an expanding Universe.-The evolution of the magnetic field via the Biermann battery process is described by a simple combination of the Maxwell-Faraday equation and the generalized Ohm's law e.g., Ref. [37]. Since we are interested in magnetic field evolution over cosmic times, we work with the Biermann battery equation in a flat expanding Universe. In this case, we find that the differential equation for the clock time evolution of the magnetic field $\mathbf{B}$ is given by

$$
\frac{\partial}{\partial t}\left(a^{2} \mathbf{B}\right)=a \nabla \times(\mathbf{u} \times \mathbf{B})-c \frac{\nabla n_{e} \times \nabla P_{e}}{e n_{e}^{2}},
$$

where $n_{e}$ and $P_{e}$ are the electron number density and pressure, respectively, $e$ is the electron charge, $\mathbf{u}$ is the peculiar velocity of the gas, and the spatial derivatives are with respect to co-moving coordinates. This equation can be reduced to the familiar form of the Biermann battery by rescaling the relevant quantities similarly to Refs. [38,39]. The resulting equation was used in describing a recent laboratory experiment of the Biermann battery [40]. Below we do not rescale the equations since $\delta_{T}$ and $\delta_{e}$ have a complicated dependence on the scale factor [25]. The term $\nabla \times(\mathbf{u} \times \mathbf{B})$, describes flux freezing, i.e., the magnetic flux through any closed contour embedded in the plasma is conserved under plasma motions. The last term is the Biermann battery term, which is proportional to the derivative with respect to time of the vorticity of the electrons; a vortexlike motion of the electrons produces a rotational electric field, and through this a magnetic field.

Consider now the Biermann term $c \nabla n_{e} \times \nabla P_{e} / e n_{e}^{2}$. The electron pressure is given by $P_{e}=n_{e} k_{B} T$, where, following [25], we have set $T_{e}=T$. Expanding the relevant quantities to linear order, i.e., $n_{e}=\bar{n}_{e}\left(1+\delta_{e}\right)$ and $T=\bar{T}\left(1+\delta_{T}\right)$, and neglecting the flux-freezing term [41], Eq. (7) can be written as

$$
\frac{\partial}{\partial t}\left(a^{2} \mathbf{B}\right)=-\frac{c k_{B} \bar{T}}{e} \nabla \delta_{e} \times \nabla \delta_{T} .
$$

Note that $n_{e}$ cancels out and the Biermann effect depends only on $\delta_{e}$. Therefore, the fact that the ionization fraction of the gas is very low $\left(\sim 10^{-4}\right)$ is not important.

The right-hand side of Eq. (8) may be written in Fourier space as

$$
\begin{aligned}
-\nabla \delta_{e} \times \nabla \delta_{T}= & \frac{1}{2} \int \frac{d^{3} k_{1}}{(2 \pi)^{3}} \frac{d^{3} k_{2}}{(2 \pi)^{3}}\left(\mathbf{k}_{1} \times \mathbf{k}_{2}\right) \\
& \times e^{i \mathbf{r}\left(\mathbf{k}_{1}+\mathbf{k}_{2}\right)}\left[\delta_{e}\left(\mathbf{k}_{1}\right) \delta_{T}\left(\mathbf{k}_{2}\right)-\delta_{e}\left(\mathbf{k}_{2}\right) \delta_{T}\left(\mathbf{k}_{1}\right)\right] .
\end{aligned}
$$

Fourier transforming both sides of Eq. (8), we then find

$$
\begin{aligned}
\frac{\partial}{\partial t}\left(a^{2} \mathbf{B}_{\mathbf{k}}\right)= & \frac{1}{2} \frac{c k_{B} \bar{T}}{e} \int \frac{d^{3} k_{1}}{(2 \pi)^{3}}\left(\mathbf{k}_{1} \times\left[\mathbf{k}-\mathbf{k}_{1}\right]\right) \\
& \times\left[\delta_{e}\left(\mathbf{k}_{1}\right) \delta_{T}\left(\mathbf{k}-\mathbf{k}_{1}\right)-\delta_{e}\left(\mathbf{k}-\mathbf{k}_{1}\right) \delta_{T}\left(\mathbf{k}_{1}\right)\right],
\end{aligned}
$$

where $\mathbf{B}_{\mathbf{k}}$ has units of $\mathrm{GMpc}^{3}$. The overdensities that appear here are complex, i.e., $\delta(k)=|\delta(k)| e^{i \phi_{k}}$, where each $\phi_{k}$ represents a random phase which is uniformly distributed over the interval 0 to $2 \pi$. The phases disappear below when we finally compute the power spectrum of the magnetic field.

The Biermann battery produces a magnetic field only if the gradients $\nabla \delta_{e}$ and $\nabla \delta_{T}$ in Eq. (8) are not parallel to each other. The equivalent condition in Fourier space is that the quantity in square brackets in Eq. (10) should be nonvanishing. The latter condition requires the ratio $\delta_{T}(\mathbf{k}) / \delta_{e}(\mathbf{k})$ to vary with scale. This is precisely where the correct treatment of the gas thermodynamics, as described in Ref. [25], is critical. As Fig. 1 shows, the ratio of temperature to density fluctuations does vary with $k$, and therefore we expect the cosmological Biermann battery to operate even within linear perturbation theory.

Let us define $\Delta_{e, T}\left(\mathbf{k}, \mathbf{k}_{1}\right)=\delta_{e}\left(\mathbf{k}_{1}\right) \delta_{T}\left(\left|\mathbf{k}-\mathbf{k}_{1}\right|\right)-$ $\delta_{e}\left(\left|\mathbf{k}-\mathbf{k}_{1}\right|\right) \delta_{T}\left(\mathbf{k}_{1}\right)$. Equation (10) then becomes

$a H \frac{\partial\left(a^{2} \mathbf{B}_{\mathbf{k}}\right)}{\partial a}=\frac{c k_{B}}{e} \int \frac{d^{3} k_{1}}{(2 \pi)^{3}} \bar{T}(t)\left(\mathbf{k}_{1} \times \mathbf{k}\right) \Delta_{e, T}\left(k, k_{1}\right)$, 
where $\partial / \partial a \equiv a H \partial / \partial t$. In this equation, only $\Delta_{e, T}$ and $\bar{T}$ depend on the time $t$ (or equivalently the scale factor $a$ ). Thus we can write Eq. (11) as

$$
\mathbf{B}_{\mathbf{k}}(a)=\int \frac{2 \pi d k_{1} \sin \theta d \theta}{(2 \pi)^{3}} \beta\left(a, k, k_{1}, \theta\right)\left(\mathbf{k}_{1} \times \mathbf{k}\right),
$$

where the quantity $\beta=\beta\left(a, k, \sqrt{k^{2}+k_{1}^{2}-2 k k_{1} \cos \theta}\right)$ satisfies

$$
a H \frac{\partial\left[a^{2} \beta\left(a, k, k_{1}\right)\right]}{\partial a}=\frac{c k_{B}}{e} \bar{T}(a) \Delta_{e, T}\left(k, k_{1}\right) .
$$

By numerically integrating Eq. (13), we can calculate the two-dimensional array of values $\beta\left(k, k_{1}\right)$ as a function of scale $a$ or redshift $z$. These $\beta$ values still include the random phases $\phi_{k}$. However, the phases are eliminated when we compute the power spectrum of the magnetic field $P_{B}$. The result is

$$
\begin{aligned}
P_{B} & \equiv\left\langle\mathbf{B}_{\mathbf{k}} \mathbf{B}_{\mathbf{k}}^{\star}\right\rangle \\
& =\frac{1}{V} \int \frac{2 \pi d k_{1} \sin \theta d \theta}{(2 \pi)^{3}}\left|\beta\left(a, k, k_{1}, \theta\right)\right|^{2}\left(k_{1} k \sin \theta\right)^{2},
\end{aligned}
$$

where $V$ is the volume.

In Fig. 2 we show $P_{B}$ as a function of wave number $k$ for different redshifts. The quantity $\sqrt{k^{3} P_{B}}$ has units of gauss. Note that the magnetic field grows most strongly on the Jeans mass scale of the baryons. This is apparent in the case

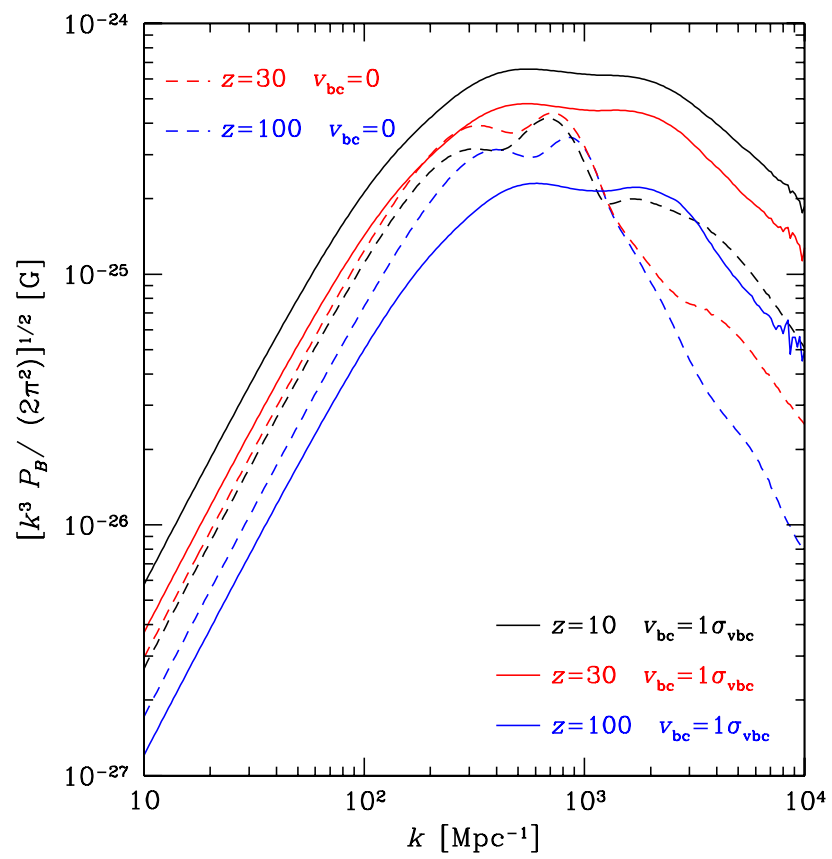

FIG. 2 (color online). Root mean square magnetic field generated by the Biermann battery as a function of wave number. Two cases are shown: no stream velocity, $v_{\mathrm{bc}}=0$ (solid lines), and a typical stream velocity, $v_{\mathrm{bc}}=1 \sigma_{\mathrm{vbc}}$ (dashed lines). Three redshifts are considered: $z=100$ (blue lines), $z=30$ (red lines), $z=10$ (black lines). of zero stream velocity, where the first peak is around $k^{-1} \sim 16 \mathrm{kpc}$ [comoving] at $z=100$, corresponding to a mass scale $\sim 7 \times 10^{4} \mathrm{M}_{\odot}$. The second peak, where the power is maximum, is associated with smaller scales $\sim 7 \mathrm{kpc}$ [comoving], which correspond to where the most dramatic variation of the ratio $\delta_{T} / \delta_{e}$ occurs (see Fig. 1). For the case of $v_{\mathrm{bc}}=1 \sigma_{\mathrm{vbc}}$, we see the inverse behavior. Here the first peak (larger scales) has more power than the second peak (smaller scales). Note that our use of linear theory is justified, since the density perturbations are still linear for scales smaller than $\sim 1000 \mathrm{Mpc}^{-1}$ [comoving] and become nonlinear only at $z<10$ (see Fig. 6 in Ref. [42]).

Discussion.-We have shown that seed magnetic fields can be produced from zero initial magnetic field on cosmological linear overdensity scales through the Biermann process. The typical field strength is $\sim 10^{-25}-10^{-24} \mathrm{G}$. These seed fields may later be amplified via nonlinear dynamo processes $[43,44]$ and are perhaps responsible for the present day magnetic fields in galaxies. Note that baryonic outflows can still contribute to the IGM magnetic field [6]. The Biermann battery mechanism requires a vortex like motion in the plasma. We have demonstrated that the spatially varying speed of sound of gas in the early Universe produces this vorticity in the residual free electrons. The process does not depend on the fraction of free electrons in the Universe but only on fluctuations in this quantity.

During reionization, the temperature of the baryons as well as temperature fluctuations will increase. This will lead to even larger magnetic fields since Eq. (8) shows that the magnetic field growth depends linearly on $\bar{T}$, and the temperature after reionization increases to $\bar{T} \rightarrow 10^{4} \mathrm{~K}$. The temperature and electron fraction fluctuations are also expected to increase substantially [45]. Thus, the magnetic field could potentially increase post-re-ionization by 4-6 orders of magnitude, bringing it close to the $10^{-18} \mathrm{G}$ estimated from observations [8-10]. This value is about 6 orders of magnitude smaller compared to other mechanisms in the literature that operate on the relevant scales (see Ref. [7] for review), but comparable to Ref. [46]. However, the evolution of $\delta_{e}$ and $\delta_{T}$ during and after reionization is model dependent. In contrast, we have shown in this Letter that, even before reionization, magnetic field can be generated as part of the linear growth of perturbations in the Universe, and that the field strength due to this process can be estimated robustly with few uncertainties.

The effect described here (following Ref. [25]) produces a vorticity in the baryonic gas on the order of $\sim 10^{-20} \mathrm{~s}^{-1}$ at $z \sim 10$ on scales $\sim 6 \mathrm{kpc}$. During reionization, as in the case of the magnetic field, the vorticity in the gas may again increase by 4-6 orders of magnitude, bringing it close to $10^{-15} \mathrm{~s}^{-1}$, which is the vorticity of the Milky Way Galaxy in the solar neighborhood. 
Future measurements of the magnetic field in the IGM and in filaments would be helpful to further clarify the role of seed magnetic fields. Already, lower bounds on the magnetic field in large scale structures [8-10,47-49] suggest that there must be a primordial seed field in the Universe. The Biermann Battery process described here, which operates through a spatially varying speed of sound, can naturally explain these seeds. Our calculation suggests that different coherent patches in the Universe with different stream velocities may have up to an order of magnitude variation in their magnetic fields. Thus, seed magnetic fields could conceivably be used in the future to study the stream velocity distribution in the Universe.

We thank Antoine Bret, Avi Loeb, and Lorenzo Sironi for useful discussions. We also thank Francesco Miniati and the anonymous referees for useful comments on the manuscript. S. N. was supported by NASA through an Einstein Postdoctoral Fellowship awarded by the Chandra X-ray Center, which is operated by the Smithsonian Astrophysical Observatory for NASA under Contract No. PF2-130096. R. N. acknowledges partial support from NASA Grant No. NNX11AE16G.

*snaoz@cfa.harvard.edu

[1] R. Beck, A. Brandenburg, D. Moss, A. Shukurov, and D. Sokoloff, Annu. Rev. Astron. Astrophys. 34, 155 (1996).

[2] L. M. Widrow, Rev. Mod. Phys. 74, 775 (2002).

[3] J.P. Vallée, New Astron. Rev. 48, 763 (2004).

[4] M. L. Bernet, F. Miniati, S. J. Lilly, P. P. Kronberg, and M. Dessauges-Zavadsky, Nature (London) 454, 302 (2008).

[5] S. Chakraborti, N. Yadav, C. Cardamone, and A. Ray, Astrophys. J. Lett. 746, L6 (2012).

[6] P. P. Kronberg, Rep. Prog. Phys. 57, 325 (1994).

[7] R. Durrer and A. Neronov, arXiv:1303.7121.

[8] A. Neronov and I. Vovk, Science 328, 73 (2010).

[9] J. Aleksić et al., Astropart. Phys. 524, A77 (2010).

[10] C. D. Dermer, M. Cavadini, S. Razzaque, J. D. Finke, J. Chiang, and B. Lott, Astrophys. J. Lett. 733, L21 (2011).

[11] D. Ryu, D. R. G. Schleicher, R.A. Treumann, C. G. Tsagas, and L. M. Widrow, Space Sci. Rev. 166, 1 (2012).

[12] A. E. Broderick, P. Chang, and C. Pfrommer, Astrophys. J. 752, 22 (2012).

[13] F. Miniati and A. Elyiv, arXiv:1208.1761.

[14] J. R. Shaw and A. Lewis, Phys. Rev. D 86, 043510 (2012).

[15] T. Kahniashvili, Y. Maravin, A. Natarajan, N. Battaglia, and A. G. Tevzadze, arXiv:1211.2769.

[16] K. L. Pandey and S. K. Sethi, Astrophys. J. 762, 15 (2013).

[17] L. Biermann, Z. Naturforsch. 5, 65 (1950).

[18] L. Mestel and I. W. Roxburgh, Astrophys. J. 136, 615 (1962).

[19] K. Subramanian, D. Narasimha, and S. M. Chitre, Mon. Not. R. Astron. Soc. 271, L15 (1994).

[20] N. Y. Gnedin, A. Ferrara, and E. G. Zweibel, Astrophys. J. 539, 505 (2000).
[21] H. Xu, B. W. O'Shea, D. C. Collins, M. L. Norman, H. Li, and S. Li, Astrophys. J. Lett. 688, L57 (2008).

[22] K. Subramanian, arXiv:0802.2804.

[23] L. M. Widrow, D. Ryu, D.R.G. Schleicher, K. Subramanian, C. G. Tsagas, and R. A. Treumann, Space Sci. Rev. 166, 37 (2012).

[24] K. Ichiki, K. Takahashi, H. Ohno, H. Hanayama, and N. Sugiyama, Science 311, 827 (2006).

[25] S. Naoz and R. Barkana, Mon. Not. R. Astron. Soc. 362, 1047 (2005)

[26] D. Tseliakhovich and C. Hirata, Phys. Rev. D 82, 083520 (2010).

[27] D. Tseliakhovich, R. Barkana, and C. Hirata, arXiv:1012.2574.

[28] A. Stacy, V. Bromm, and A. Loeb, Astrophys. J. Lett. 730, L1 (2011).

[29] T. H. Greif, S. C. O. Glover, V. Bromm, and R. S. Klessen, Astrophys. J. 716, 510 (2010).

[30] E. Visbal, R. Barkana, A. Fialkov, D. Tseliakhovich, and C. Hirata, arXiv:1201.1005.

[31] S. Naoz, N. Yoshida, and N. Y. Gnedin, Astrophys. J. 747, 128 (2012).

[32] R. M. O'Leary and M. McQuinn, arXiv:1204.1344.

[33] S. Naoz, N. Yoshida, and N. Y. Gnedin, Astrophys. J. 763, 27 (2013).

[34] E. Komatsu, J. Dunkley, M. R. Nolta, C. L. Bennett, B. Gold, G. Hinshaw, N. Jarosik, D. Larson, M. Limon, L. Page, D. N. Spergel, M. Halpern, R. S. Hill, A. Kogut, S. S. Meyer, G. S. Tucker, J. L. Weiland, E. Wollack, and E. L. Wright, Astrophys. J. Suppl. Ser. 180, 330 (2009).

[35] S. Naoz and R. Barkana, Mon. Not. R. Astron. Soc. 377, 667 (2007).

[36] R. Barkana and A. Loeb, Mon. Not. R. Astron. Soc. 363, L36 (2005).

[37] R. M. Kulsrud, Plasma Physics for Astrophysics, Princeton Series in Astrophysics (Princeton University Press, Princeton, N.J., 2005).

[38] C.P. Dettmann, N. E. Frankel, and V. Kowalenko, Phys. Rev. D 48, 5655 (1993).

[39] K. Subramanian, Astron. Nachr. 331, 110 (2010).

[40] G. Gregori et al., Nature (London) 481, 480 (2012).

[41] Approximating $u \sim c_{s}^{2} \delta_{e} k /(H a)$, we find that for $10^{-24} \mathrm{G}$ the flux freezing term is still much smaller $\left(\$ 10^{4-6}\right)$ than the Biermann term, and thus we neglect it for the future calculations, e.g., [37].

[42] R. Barkana and A. Loeb, Phys. Rev. 349, 125 (2001).

[43] K. Subramanian and A. Brandenburg, Phys. Rev. Lett. 93, 205001 (2004).

[44] S. Sur, D. R. G. Schleicher, R. Banerjee, C. Federrath, and R. S. Klessen, Astrophys. J. Lett. 721, L134 (2010).

[45] J. R. Pritchard and S. R. Furlanetto, Mon. Not. R. Astron. Soc. 376, 1680 (2007).

[46] F. Miniati and A. R. Bell, Astrophys. J. 729, 73 (2011).

[47] Y. Xu, P.P. Kronberg, S. Habib, and Q. W. Dufton, Astrophys. J. 637, 19 (2006).

[48] D. Ryu, H. Kang, and P. L. Biermann, AAP 335, 19 (1998).

[49] D. Ryu, H. Kang, J. Cho, and S. Das, Science 320, 909 (2008). 